\title{
Diseño y Proyecto, competencia transversal del Grado en Gestión y Administración Pública
}

\author{
Puertas, Rosa ${ }^{a}$, Giménez-Chornet, Vicent ${ }^{\mathrm{b}}$, Orea, Guadalupe ${ }^{\text {a }}$, González, Silvia ${ }^{\text {a }}$ \\ a) Departamento de Economía y Ciencias Sociales, rpuertas@esp.upv.es; lupeorea@gmail.com; silgonde@upv.es \\ b) Departamento de Comunicación Audiovisual, Documentación e Historia del Arte. Facultad de Administración y \\ Dirección de Empresas. Universitat Politècnica de València vigicho@har.upv.es
}

\begin{abstract}
The strategic plan of the UPV2020 includes the correct accreditation of the transversal competences (TC) defined by the Universitat Politècnica de València (UPV). The aim is to achieve innovative educational models based on the transmission of knowledge linked to competences that make it easier for graduates to enter the world of work. The aim of this research is to analyse the implementation of the transversal competence 5, Design and Project, in the Grade of Management and Public Administration (GAP) of the UPV, in order to achieve a system of coordination between the subjects that are control points (PC). A review of the learning activities used and the chosen evaluation system will be carried out to evidence the acquisition of competence. The analysis of feedback between teachers will facilitate the analysis and collection of information.
\end{abstract}

The results will allow to detect the degree of adequacy of the same one so much to level of imparted matter, as of acquisition of the same one on the part of the student. In addition, lines of improvement will be established to ensure consistency between the object and evaluate and the procedure chosen.

Keywords: se Design and Project; evaluation; transversal competence; learning activities

\section{Resumen}

El plan estratégico de la UPV2020 incluye la correcta acreditación de las competencias transversales (CT) definidas por la Universitat Politècnica de València (UPV). Se trata de lograr modelos educativos innovadores basados en la transmisión de conocimientos ligados a competencias que faciliten a los egresados la inserción en el mundo laboral. El objetivo de esta investigación es analizar la implementación de la competencia transversal 5, Diseño y Proyecto, en el Grado de Gestión y Administración Pública (GAP) de la UPV, para lograr un sistema de coordinación entre las asignaturas que son punto de control (PC). Se realizará una revisión de las actividades de aprendizaje utilizadas y del sistema de evaluación elegido para evidenciar la adquisición de la competencia. El análisis del feedback entre profesores facilitará el análisis y la recogida de información. 
Los resultados permitirán detectar el grado de adecuación de la misma tanto a nivel de materia impartida, como de adquisición de la misma por parte del estudiante. Además, se establecerán las líneas de mejora que garanticen la coherencia entre el objeto e evaluar y el procedimiento elegido.

Palabras clave: Diseño y Proyecto; evaluación; competencia transversal; actividades de aprendizaje

\section{Introducción}

La palabra "globalización" ha venido para instaurarse en nuestra sociedad a todos los niveles, no solo nivel empresarial sino también educativo. Las universidades necesitan adecuar sus programas de formación a las necesidades del trabajo futuro, donde las competencias de creatividad, innovación, comunicación, trabajo en equipo, entre otras, están adquiriendo una relevancia equiparable a las competencias profesionales implementadas en todos los grados universitarios. Según Riera (2017) se trata de competencias genéricas presentes en las relaciones profesionales estrechamente relacionadas con las aptitudes, los rasgos de la personalidad, los conocimientos adquiridos y los valores.

Gaxiola y Patrón (2017) consideran que la formación académica profesional está garantizada en los planes de estudio tradicionales, pero ésta es insuficiente para asegurar al egresado la correcta integración en el mundo laboral. Existen competencias o destrezas no técnicas que están escalando posiciones en la demanda del mercado laboral, de ahí que los centros de educación superior tienen la obligación de adaptarse para garantizar una formación completa, competitiva y flexible en un entorno cambiante. Estas competencias transversales (CT) no son propias de un puesto de trabajo concreto como lo serían las técnicas, sino que están ligadas a las cualidades necesarias para realizar correctamente las tareas profesionales encomendadas.

Según el último informe del Foro Económico Mundial, titulado The Future of Jobs, las 10 principales habilidades que deberían reunir los futuros trabajadores deben revisarse periódicamente para enfrentarse a los continuos avances tecnológicos (World Economic Forum, 2017). Se tiene que estar preparado para poder realizar un aprendizaje continuo, que facilite la adaptación de las competencias aprendidas a las exigencias del mercado laboral. Así en la Tabla 1 se muestra la demanda de los principales empleadores mundiales en dos momentos distintos del tiempo, y que deberían reunir los trabajadores del futuro.

Educar en competencias supone también un reto para el profesorado, que debe de adquirir habilidades, como el uso de las TIC, para desenvolverse en el aula y en las nuevas plataformas digitales docentes (Belando-Montoro, 2014). Esta competencia digital es un valor añadido en los docentes requerida por una sociedad más tecnológica (López Belmonte, Pozo Sánchez, Morales Cevallos, López Meneses, 2019). 
Tabla 1. Principales competencias del futuro trabajador

\begin{tabular}{|c|c|c|c|}
\hline & en 2015 & & en 2020 \\
\hline 1. & Resolución de problemas complejos & 1. & Resolución de problemas complejos \\
\hline 2. & Coordinación con otros & 2. & Pensamiento crítico \\
\hline 3. & Gestión de personal & 3. & Creatividad \\
\hline 4. & Pensamiento crítico & 4. & Gestión de personal \\
\hline 5. & Negociación & 5. & Coordinación con otros \\
\hline 6. & Control de calidad & 6. & Inteligencia emocional \\
\hline 7. & Orientación del servicio & 7. & Juicio y toma de decisiones \\
\hline 8. & Juicio y toma de decisiones & 8. & Orientación del servicio \\
\hline 9. & Escucha activa & 9. & Negociación \\
\hline 10. & Creatividad & 10. & Flexibilidad cognitiva \\
\hline
\end{tabular}

Fuente: Elaboración propia. Datos Future of Jobs. World Economic Forum, 2017.

La correcta evaluación de las CT es clave para la adecuación del candidato al puesto de trabajo ofertado, de ahí la importancia de implementar herramientas de análisis y valoración apropiadas. En este marco conceptual, la investigación se centra en el sistema de evaluación de la CT 5, Diseño y proyecto, en aquellas asignaturas que son punto de control (PC) en el Grado de Gestión y Administración Pública (GAP) impartido en la Universitat Politècnica de València (UPV). Se realizará un análisis previo de las asignaturas en cuestión para poder valorar las actividades de aprendizaje que facilitan al alumno la adquisición de dicha competencia.

El artículo se estructura en las siguientes secciones. En la siguiente sección se presenta el proyecto de CTs de la UPV, centrándose en la CT 5 y en las rúbricas generales publicadas para su evaluación. En la sección tercera se presentan las asignaturas que la evalúan para conocer sus características propias y como trasladan el aprendizaje de la misma. En la sección cuarta, se presentan los resultados obtenidos en los últimos años. Por último, en la sección quinta se resumen las principales conclusiones del estudio.

\section{Competencias transversales de la UPV: Diseño y Proyecto (CT5)}

A iniciativa del Vicerrectorado de Estudios, Calidad y Acreditación de la UPV se ha implementado en todos los títulos oficiales impartidos en este campus 13 CTs que tratarán de cuantificar la capacidad de los egresados, proporcionando una medida de su valor 
añadido para otorgarles un atractivo diferenciador frente a sus competidores. A continuación, se muestra una breve descripción de todas ellas.

CT1. Comprensión e integración. Demostrar la comprensión y la integración del conocimiento tanto de la especialización propia como en otros contextos más amplios.

CT2. Aplicación y pensamiento práctico. Aplicar los conocimientos teóricos y establecer el proceso a seguir para alcanzar determinados objetivos, llevar a cabo experimentos y analizar e interpretar datos para extraer conclusiones.

CT3. Análisis y resolución de problemas. Analizar y resolver problemas de forma efectiva, identificando y definiendo los elementos significativos que los constituyen.

CT4. Innovación, creatividad y emprendimiento. Innovar para responder satisfactoriamente y de forma original a las necesidades y demandas personales, organizativas y sociales con una actitud emprendedora.

CT5. Diseño y proyecto. Diseñar, dirigir y evaluar una idea de manera eficaz hasta concretarla en un proyecto.

CT6. Trabajo en equipo y liderazgo. Trabajar y liderar equipos de forma efectiva para la consecución de objetivos comunes, contribuyendo al desarrollo personal y profesional de los mismos.

CT7. Responsabilidad ética, medioambiental y profesional. Actuar con responsabilidad ética, medioambiental y profesional ante uno mismo y los demás.

CT8. Comunicación efectiva. Comunicarse de manera efectiva, tanto de forma oral como escrita, utilizando adecuadamente los recursos necesarios y adaptándose a las características de la situación y de la audiencia.

CT9. Pensamiento crítico. Desarrollar un pensamiento crítico interesándose por los fundamentos en los que se asientan las ideas, acciones y juicios, tanto propios como ajenos.

CT10. Conocimiento de problemas contemporáneos. Identificar e interpretar los problemas contemporáneos en su campo de especialización, así como en otros campos del conocimiento, prestando especial atención a los aspectos relacionados con la sostenibilidad.

CT11. Aprendizaje permanente. Utilizar el aprendizaje de manera estratégica, autónoma y flexible, a lo largo de toda la vida, en función del objetivo perseguido.

CT12. Planificación y gestión del tiempo. Planificar adecuadamente el tiempo disponible y programar las actividades necesarias para alcanzar los objetivos, tanto académico profesionales como personales. 
CT13. Instrumental específico. Utilizar las técnicas, las habilidades y las herramientas actualizadas necesarias para la práctica de la profesión.

Se trata de habilidades caracterizadas por favorecer la formación integral, además de ser transferibles en diversos contextos académicos, laborales y personales. Deben ser versátiles para ser capaces de responder en distintos entornos y, por supuesto, evaluables con objeto de poder acreditar el nivel alcanzado por los egresados.

La evaluación de las mismas se realiza a través del seguimiento evolutivo de determinadas asignaturas seleccionadas como PC a lo largo del grado. Cada una de estas asignaturas debe diseñar actividades de aprendizaje y evaluación para desarrollar y, posteriormente, evidenciar la adquisición de la CT, siendo capaces de poder recoger evidencias de su trabajo y evaluación.

A continuación, nos vamos a centrar en la CT5, el desarrollo de esta competencia favorece que el estudiante aprenda haciendo e integrando conocimientos y habilidades de diferentes ámbitos disciplinares, desarrollando habilidades intelectuales de alto nivel, promoviendo el aprendizaje y trabajo autónomo, el trabajo en equipo y la autoevaluación Para ello, la UPV recomienda una serie de metodologías e instrumentos de evaluación para cada una de las 13 CT. Concretamente, para la CT5 se recomienda lo siguiente:

- Metodología de aprendizaje:

- Actividades de grupales

- Exposiciones orales

- Lección magistral

- Lecturas

- Proyectos

- Redacción de informes

- Tutorías planificadas

- Sistemas de evaluación:

- Autoevaluación

- Evaluación entre iguales

- Exposición oral

- Observación

- Portafolio

- Prueba escrita de respuesta abierta

- Rúbricas

La UPV ha diseñado unas rúbricas de orientación para la correcta evaluación de todas las CT. Todas ellas deberán ser evaluadas dos veces en el primer ciclo del grado (Nivel 1) y otras dos en segundo (Nivel 2). Concretamente en la CT5, al finalizar el segundo curso del grado el alumno tendrá que ser capaz de diseñar un proyecto de trabajo sin llegar a su ejecución, es decir, justificar su necesidad, establecer objetivos, proponer acciones para su consecución, asignar plazos de ejecución e identificar riesgos. En el nivel 2 ( $4^{\circ}$ curso) 
se requerirá que sean capaces de planificar proyectos en colaboración con otros en situaciones poco estructuradas, prever incidencias y riesgos. La calificación de la misma en los dos niveles oscila entre A (excelente) y D (no alcanzado), según el grado de consecución de la misma.

\section{Puntos de control de la CT 5}

Actualmente, por un error en la matriz de CT, la competencia Diseño y Proyecto sólo se está evaluando en el segundo ciclo por las asignaturas Gestión de Documento Electrónico y Control de Costes en los sistemas de Salud y Servicios Sociales Públicos. El próximo curso académico se evaluará en el nivel 1 a través de las asignaturas Economía Política y Economía del Sector Público.

La competencia Diseño y Proyecto reúne en sí otras competencias relacionadas con la gestión de proyectos, cuyo desarrollo en el alumnado puede contribuir en su vida laborar al logro de los objetivos estratégicos de una organización (Cha, Eunice MaytorenaSanchez, 2019). En el sector público se requiere que existan mecanismos que aseguren el desarrollo de competencias de sus empleados para promover la innovación, al trabajar en proyectos y otras tareas de resolución de problemas (Medina, 2017).

\subsection{Gestión de Documento Electrónico}

Se trata de una asignatura optativa transversal que se imparte en el tercer curso del grado en GAP de la UPV, y tiene una carga académica de 4,5 créditos. Está asignada al departamento de Comunicación Audiovisual, Documentación e Historia del Arte, concretamente al área de Biblioteconomía y Documentación.

El objetivo de la asignatura es trasladar al alumno la importancia de la Administración Electrónica para que conozca los fundamentos de la gestión de documentos digitales, realizando para ello prácticas relacionadas con portales web de administraciones públicas, metadatos, procedimientos administrativos y expedientes electrónicos. Consta de 4 unidades didácticas que facilitarán la adquisición de las competencias técnicas: Nuevo paradigma en la documentación electrónica, características del documento electrónico, política de gestión de documentos electrónicos y preservación digital. Además de la CT5, tiene asignada la CT11, Aprendizaje permanente.

Los docentes de esta asignatura han establecido que la adquisición de esta competencia se realizará a través del análisis de un procedimiento administrativo electrónico. El alumno tendrá que elegir un trámite en una de las administraciones a la que tenga acceso con su certificado digital, para estudiar tanto el mapa de documentos como los elementos de la tramitación electrónica. La evaluación de esta CT se materializará mediante la redacción de un trabajo y su posterior exposición en el aula, valorándose positivamente los elementos de reflexión propia. 
El trabajo ligado a esta CT es obligatorio para obtener la nota global de la signatura, el $25 \%$ de la misma está ligado a la nota que se asignará a la CT5. En caso de suspender la asignatura se considerará no superada la CT.

\subsection{Control de Costes en los Sistemas de Salud y Servicios Sociales Públicos}

Es una asignatura optativa de la intensificación de Dirección y Gerencia Económica que se imparte en el cuarto curso del grado en GAP de la UPV y tiene una carga de 4,5 créditos. Está asignada al departamento de Economía y Ciencias Sociales, concretamente al área de Economía Aplicada.

Esta asignatura facilita al alumno las bases de la gestión de costes en el sistema de salud y en los servicios sociales para personas con dependencia. Con este objetivo, en primer lugar, se estudia la organización de los sistemas públicos de bienestar social y salud, y en segundo lugar su financiación, así como los problemas que deben afrontar en la coyuntura económica actual. Al finalizar la asignatura el alumno conocerá la forma de medición y evaluación de los costes, pudiendo discernir las medidas de control de las administraciones públicas y los centros asistenciales. Consta de 3 unidades didácticas que facilitan la adquisición de las competencias técnicas propias de esta asignatura: Introducción a la económica de la salud, Costes en servicios de salud y Costes en servicios sociales. Además de la CT5 tiene asignada la CT7, Responsabilidad ética, medioambiental y profesional.

La adquisición de la CT5 se propone mediante el diseño y elaboración de un proyecto relacionado con los temas impartidos en la asignatura. Este será elaborado de manera grupal y presentado en las últimas semanas del cuatrimestre. Se asignará una nota global al grupo y constituye el $20 \%$ de la nota global de la asignatura además de la valoración de la CT5.

\section{Evaluación de la CT5}

El registro de resultados de la evaluación de las CT en la UPV está disponible tan sólo el correspondiente a los tres últimos cursos académicos (2015-16, 2016-17 y 2017-18). En los dos primeros se obtuvo evaluación tanto en el nivel 1 como en el 2, sin embargo, en 2017-18 tan sólo ha sido evaluada en el nivel 2, de ahí que para el próximo curso (20192020) haya sido asignada como PC a dos asignaturas del primer ciclo del grado de GAP (Tabla 2).

Tabla 2. Evaluación de la adquisición de la CT 5 en el grado de GAP

\begin{tabular}{c|cccc|cccc}
\hline & \multicolumn{5}{c|}{ Nivel 1 } & \multicolumn{5}{c}{ Nivel 2 } \\
\cline { 2 - 9 } \multicolumn{2}{c}{ A } & B & C & D & A & B & C & D \\
\hline \multirow{2}{*}{$\mathbf{2 0 1 5 - 1 6}$} & 3 & 8 & 49 & 24 & 0 & 28 & 0 & 0 \\
& $3,5 \%$ & $9,5 \%$ & $58,3 \%$ & $28,5 \%$ & & $100 \%$ & & \\
\hline
\end{tabular}




\begin{tabular}{c|cccc|cccc}
\hline $\mathbf{2 0 1 6 - 1 7}$ & 5 & 27 & 30 & 0 & 1 & 31 & 0 & 0 \\
& $8,1 \%$ & $43,5 \%$ & $48,4 \%$ & & $3,1 \%$ & $96,9 \%$ & & \\
\hline \multirow{2}{*}{$2017-18$} & - & - & - & - & 8 & 13 & 1 & 0 \\
& & & & & $36,4 \%$ & $59,1 \%$ & $4,5 \%$ & 0 \\
\hline
\end{tabular}

Fuente: Elaboración propia a partir de datos facilitados por la UPV.

La información disponible es escasa debido a que la evaluación de las CT en la UPV tan sólo es obligatoria desde el curso académico 2015-2016. Sin embargo, se pueden extraer algunas conclusiones. Destaca que en el nivel 2 los alumnos se hayan más implicados en el proyecto, como corrobora las calificaciones obtenidas. La totalidad de los alumnos evaluados han superado esta competencia con una nota de A (excelente) y B (adecuado), mientras que en el nivel 1 la calificación está más repartida.

Se aprecia una gran dispersión en el nivel 1, casi el 50\% obtiene una nota de C, indicando la necesidad del alumno de adquirir más formación que el alumno en dicha competencia, es decir, se haya todavía en desarrollo.

\section{Conclusiones}

El sistema educativo actual se halla inmerso en un proceso de cambio y desarrollo para adaptarse a las necesidades del mercado laboral. Las competencias profesionales técnicas deben ser complementadas con transversales que valoren las actitudes de los egresados y su grado de adaptación a los puestos ofertados.

Las CT deben ser cambiantes para que crezcan y se desarrollen de forma paralela a los cambios inherentes en el trabajo futuro. La valoración de estas competencias otorga a los estudiantes un valor añadido y, por tanto, mayor competitividad para enfrentarse al tejido empresarial internacional en las mejores condiciones.

Este artículo ha tratado de analizar el sistema de evaluación de la CT5, con objeto de constituir un punto de partida para ir completando con los resultados de los siguientes cursos académicos y poder valorar de forma conjunta cambios en los sistemas de evaluación de la misma. En la actualidad, no se dispone de información suficiente para detectar si lo realizado hasta la fecha es insuficiente. Los docentes han manifestado su conformidad y satisfacción con las actividades de aprendizaje realizadas para su consecución.

\section{Bibliografía}

BELANDO-MONTORO, M. R. (2014). Formación permanente del profesorado. Algunos recursos TIC para la docencia universitaria. Revista Iberoamericana de Educación, vol. 65, issue 1, p. 1-11. DOI: https://doi.org/10.35362/rie651324 
CHA, J. y MAYTORENA-SANCHEZ, E. (2019). Prioritising project management competences across the software project life cycle. International Journal of Managing Projects in Business, preprint. https://doi.org/10.1108/IJMPB-11-2017-0145

GAXIOLA, J.A. y PATRÓN, D. I. (2017). Formación para el trabajo a través de competencias transversales. IV Congreso Internacional sobre Aprendizaje, Innovación y Competitividad, p. 492-496. Recuperado de https://www.researchgate.net/publication/320038683

LÓPEZ, J., POZO, S., MORALES, M. B y LÓPEZ, E. (2019). Competencia digital de futuros docentes para efectuar un proceso de enseñanza y aprendizaje mediante realidad virtual. Edutec. Revista electrónica de tecnología Educativa, vol. 67, p. 1-15. DOI 10.21556/edutec.2019.67.1327

MEDINA, R. y MEDINA, A. (2017). Managing competence and learning in knowledgeintensive, project-intensive organizations: A case study of a public organization. International Journal of Managing Projects in Business, vol. 10, issue 3, p. 505-526, https://doi.org/10.1108/IJMPB-04-2016-0032

RIERA, M. (2017). El crecimiento profesional derivado de las competencias transversales La capacidad de negociación. Harvard Deusto Business Review, vol. 267, p. 54-60.

WORLD ECONOMIC FORUM (2017). The Future of Jobs. Retrieved 8 May 2017, from http://reports.weforum.org/future-of-jobs-2016/ 